\title{
Numerical study on unstable surfaces of oblique detonations
}

\author{
Hong Hui Teng ${ }^{1} \dagger$, Zong Lin Jiang ${ }^{1}$ \\ and Hoi Dick $\mathrm{Ng}^{2}$ \\ ${ }^{1}$ State Key Lab of High Temperature Gas Dynamics, Institute of Mechanics, Chinese Academy \\ of Sciences, Beijing, 100190, China \\ ${ }^{2}$ Department of Mechanical and Industrial Engineering, Concordia University, Montreal, QC, \\ H3G 1M8, Canada \\ (Received ?; revised ?; accepted ?. - To be entered by editorial office)
}

In this study, the onset of cellular structure on oblique detonation surfaces is investigated numerically using a one-step irreversible Arrhenius reaction kinetic model. Two types of oblique detonations are observed from the simulations. One is weakly unstable characterized by the existence of a planar surface, and the other is strongly unstable characterized by the immediate formation of the cellular structure. It is found that a high degree of overdrive suppresses the formation of cellular structures as confirmed by the results of many previous studies. However, the present investigation demonstrates that cellular structures also appear with degree of overdrive of 2.06 and 2.37, values much higher than $\sim 1.8$ suggested previously in literature for the critical value defining the instability boundary of oblique detonations. This contradiction could be explained by the low and possibly insufficient resolution used in previously published results. Hence, simulations with different grid sizes are also performed to examine the effect of resolution on the numerical solutions. Using the present results, analysis also shows that although the characteristic lengths of unstable surfaces are different when the incident Mach number changes, these length scales are proportional to tangential velocities. Hence, the interior time determined by the overdrive degree is identified, and its limitation as the instability parameter is discussed.

Key words:

\section{Introduction}

Detonation waves have been studied for many years due to their potential applications in hypersonic propulsion systems, see Kailasanath (2003); Roy et al. (2004). Among those is the use of oblique detonation wave in continuous detonation wave engines and ram accelerators (Nettleton 2000). However, there remain several challenges in the development of oblique detonation-based propulsion systems, among which is the required formation of a stationary oblique structure in a supersonic flow. Hence, it is still of great interest to study the basic structure of oblique detonation waves in order to further develop its application.

In early research, the oblique detonation wave analysis was simplified into an oblique shock wave followed by an instantaneous post-shock heat release. Later in the numerical

$\dagger$ Email address for correspondence: hhteng@imech.ac.cn 
simulation performed by Li et al. (1994), the multi-dimensional oblique detonation structure was found to be composed of a non-reactive oblique shock, an induction region, a set of deflagration waves, and an oblique detonation surface. The onset or transition of the oblique detonation originates abruptly from the location of this multi-wave configuration ( $\lambda$-structure) in the vicinity of the wedge beginning. This structure was verified experimentally by Viguier et al. (1997), and most work later focuses on the characteristics of this kind of structure, e.g. Fusina et al. (2005); Choi et al. (2009). However, the work by Vlasenko \& Sabelnikov (1995) pointed out that the shock to detonation transition may occur smoothly by an arc shock, rather than occurring in a multi-wave interaction point. In addition, Papalexandris (2000) demonstrated that wedge angles may influence the transition type. Figueira da Silva \& Deshaies (2000) studied the transition distance, and indicated that the smooth transition appeared when the induction time became trivial in total chemical reaction time. Recently, Teng \& Jiang (2012) compared results with various chemical and gas dynamic parameters, and demonstrated that the transition type was controlled by the difference of oblique shock and detonation angles.

Aforementioned studies have focused mainly on the shock to detonation transition, i.e., how the oblique detonation initiates. However, the study on the oblique detonation surface, i.e., how the cellular detonation structures form and evolve, has not been studied systemically. Cellular surfaces with triple points are observed on the oblique detonations experimentally by Viguier et al. (1999). In numerical simulation, combustible mixtures with large value of activation energy are found to be more susceptible to triple point formation (Papalexandris 2000). Choi et al. (2007) show some small scale structures with several activation energies, and their result indicates that these structures cannot be captured without enough numerical resolution. However, the above mentioned cellular structures are featured mainly by a single group of triple points traveling upstream, different from normal detonations with one pair of triple points traveling in opposite direction. Recently, another group of triple points is revealed in the case when a detonation wave extends long enough downstream, for instance, see Gui et al. (2011); Verreault et al. (2013).

Although recent studies set up the connection between the oblique and normal detonation cellular structures, it remains unclear whether oblique detonations have similar instability characteristics as normal detonations. Oblique detonations are overdriven and hence, Grismer \& Powers (1996) introduced the overdrive degree as one controlling parameter to define stability boundaries for oblique detonations. Their results show that a high overdrive degree suppresses the oblique detonation instability, similar to normal detonations (Ng \& Zhang 2012). In contrast, Choi et al. (2007) studied the oblique detonation with different values of activation energy, and observed unstable surfaces even the degree of overdrive is very high. More recently, Verreault et al. (2013) studied the triple point formation, and the overdrive degree defining the instability boundary is found to be just slightly higher than that of one-dimensional normal pulsating detonations (Lee \& Stewart 1990; Bourlioux et al. 1991; Sharpe 1997). Despite the fact that the overdrive degree provides a key parameter, it is still unclear how the oblique surface becomes unstable, and what causes the discrepancy of the stability boundary of overdrive between the oblique and normal detonations.

In this study, the formation and evolution of cellular oblique detonation structures are investigated using numerical simulations. In literature, contradictions appear between numerical results obtained by different researchers. One of the main reasons is often due to the use of inadequate numerical resolution. Hence, an alternative numerical procedure is proposed in this study to ensure the same level of grid resolution is obtained for different cases with different conditions, i.e., degree of overdrive. This is achieved by 


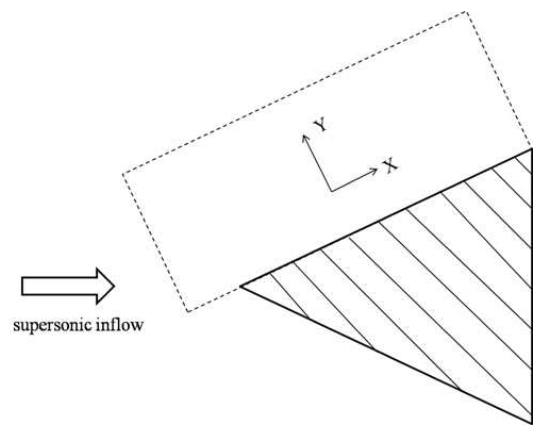

FIGURE 1. Schematic of wedge-induced oblique detonations in the combustible gas mixtures.

calibrating the pre-exponential factor in the one-step reaction model to adjust the length scale of the corresponding overdriven detonation according to a pre-specified resolution. A resolution study is also performed to verify the chosen resolution for sufficient numerical accuracy. The effect of overdrive on the dynamics of oblique detonations is then analyzed by varying the wedge angle and incident Mach number. In this work, an interior time scale is proposed to characterize the instability. Its limitation as the instability parameter is also discussed.

\section{Numerical methods}

A schematic of the oblique detonation wave induced by the wedge in a combustible gas mixture is shown in Fig. 1. An oblique shock wave forms first and subsequently, if the post-shock temperature is sufficiently high, an exothermic chemical reaction is initiated and induces an oblique detonation. For convenience, the coordinate is rotated to the direction along the wedge surface, and simulations are carried out in region enclosed by the dashed line. Similar to previous numerical studies on oblique detonations (Li et al. 1993; Grismer \& Powers 1996; Papalexandris 2000; Choi et al. 2007; Gui et al. 2011; Teng \& Jiang 2012; Verreault et al. 2013), the present study is also based on the inviscid assumption. In the present study, non-dimensional Euler equations with one-step irreversible Arrhenius kinetic model are used as the governing equations, i.e.:

$$
\begin{gathered}
\frac{\partial U}{\partial t}+\frac{\partial F}{\partial x}+\frac{\partial G}{\partial y}+S=0 \\
U=\left[\begin{array}{c}
\rho \\
\rho u \\
\rho v \\
\rho e \\
\rho \lambda
\end{array}\right], \quad F=\left[\begin{array}{c}
\rho u \\
\rho u^{2}+p \\
\rho u v \\
\rho u(e+p) \\
\rho u \lambda
\end{array}\right], \quad G=\left[\begin{array}{c}
\rho v \\
\rho u v \\
\rho v^{2}+p \\
\rho v(e+p) \\
\rho v \lambda
\end{array}\right], \quad S=\left[\begin{array}{c}
0 \\
0 \\
0 \\
0 \\
\rho \dot{\omega}
\end{array}\right],
\end{gathered}
$$

with

$$
\begin{gathered}
e=\frac{p}{(\gamma-1) \rho}+\frac{1}{2}\left(u^{2}+v^{2}\right)-\lambda q, \\
p=\rho T \\
\dot{\omega}=k(1-\lambda) \exp \left(-\frac{E_{a}}{T}\right),
\end{gathered}
$$


where $\rho, u, v, p, e$ denote the density, velocities in $x$ - and $y$ - direction, pressure and total energy, respectively. For the chemical reaction, $\lambda$ is the reaction progress variable and $q$ is the amount of heat release. The reaction is controlled by the activation energy $E_{a}$ and preexponential factor $k$. The latter is chosen to define the spatial and temporal scales. In this study, the dimensionless parameters are fixed with the values $q=50, E_{a}=50$, and $\gamma=$ 1.20 often used in previous studies of both oblique and normal one-dimensional pulsating detonations (Bourlioux \& Majda 1992; Grismer \& Powers 1996; Short \& Stewart 1998; Papalexandris 2000; Verreault et al. 2013). The governing equations are discretized on Cartesian orthogonal uniform grids and solved with the MUSCL-Hancock scheme with Strang's splitting.The MUSCL-Hancock scheme is formally a second-order extension to the Godunov's first order upwind method by constructing the Riemann problem on the inter-cell boundary. For one spatial direction, the reconstruction has the following form:

$$
\left.\begin{array}{l}
\bar{U}_{i}^{L}=U_{i}^{L}+\frac{\Delta t}{2 \Delta x}\left[F\left(U_{i}^{L}\right)-F\left(U_{i}^{R}\right)\right] \\
\bar{U}_{i}^{R}=U_{i}^{R}+\frac{\Delta t}{2 \Delta x}\left[F\left(U_{i}^{L}\right)-F\left(U_{i}^{R}\right)\right]
\end{array}\right\}
$$

and these advanced values are then used as the input for a Riemann solver at each cell interface. The scheme is made total variation diminishing (TVD) with the use of slope limiter MINBEE, which reduces the spatial accuracy to at most first-order to prevent non-physical oscillations. In this study, the HLLC solver is used for the Riemann problem. For more details about the scheme and solver, see Toro (1999).

\section{Results and Discussion}

\subsection{Characteristics of oblique detonation surfaces}

Due to the fact that oblique detonation is generally overdriven, there are essentially two challenges introduced in its numerical simulation. First, it is difficult to guarantee enough numerical grids if the resolution is specified in the reaction zone of an unsupported Zel'dovich-von Neumann-Döring (ZND) detonation. For example, when a ZND detonation becomes overdriven with an overdrive degree of 1.59, the grid resolution in the half-reaction zone changes from 128 to 13 (Verreault et al. 2013). As a result, the actual resolution used in the oblique detonation simulation should be defined relative to the corresponding overdriven detonation. On a computational domain with a fixed number of Cartesian grids, the spatial resolution is dependent on the pre-exponential factor $k$ of the reaction model. Moreover, the variation of wedge angle or incident Mach number induces a different overdrive degree, which in turn changes the spatial scale. Hence, results with the same $k$ have actually different resolutions. In this study, a computationally feasible domain of 5000 grid points in $x$-direction and 700 to 1000 grid points in $y$-direction is used. Unless specified, a fixed grid size of 0.010 was chosen in both $x$ - and $y$-directions so that the computational domain is sufficiently large to capture the entire flow field of interest. The pre-exponential factor $k$ is adjusted to obtain the reaction scale so as to give a numerical resolution of 20 points in the half-reaction zone $l_{1 / 2}$ (i.e., location where the reaction progress variable $\lambda=0.5$ ) of the corresponding overdriven detonation on the same grid domain for all simulation cases. To obtain the overdrive degree, the oblique detonation angle $\beta$ needs to be first calculated given the incident Mach number $M$ and wedge angle $\theta$, whose implicit form derives from detonation shock polar relation: 


$$
\frac{\tan \beta}{\tan (\beta-\theta)}=\frac{(\gamma+1) M^{2} \sin ^{2} \beta}{\gamma M^{2} \sin ^{2} \beta+1-\sqrt{\left(M^{2} \sin ^{2} \beta-1\right)^{2}-2\left(\gamma^{2}-1\right) M^{2} \sin ^{2} \beta \cdot q}} .
$$

And the corresponding overdrive degree is defined by:

$$
f=\left(\frac{M \sin \beta}{M_{C J}}\right)^{2},
$$

where $M_{C J}$ denotes the Mach number of the Chapman-Jouguet (CJ) detonation for a given $q$ and $\gamma$. The steady overdriven ZND structure with the overdrive degree $f$ calculated in Eq. 3.2 is then computed to determine the value of the pre-exponential factor $k$ of the reaction model so that the chemical length scale $l_{1 / 2}$ is covered with the chosen number of grids. The final $k$ can then be used in initializing the unsteady oblique detonation simulation.

It is worth noting that a resolution of 20 points per half reaction zone length, $l_{1 / 2}$, of the detonation wave is often suggested as a minimum to capture the longitudinal instability in one-dimensional pulsating detonations. This applies to cases with a high degree of overdrive factor or moderate activation energy; or when seeking only a reasonable estimation of the stability boundary using numerical simulations (Grismer \& Powers 1996; Bourlioux et al. 1991; Hwang et al. 2000; Sharpe \& Falle 1999). For sufficiently regular detonation cells as for the case with high degree of overdrive, Bourlioux \& Majda (1992) simulated detonation cells using a similar grid resolution of about 20 points $/ l_{1 / 2}$ and agreement was obtained between computed detonation cell sizes and predictions from stability theories. The later work by Choi et al. (2008) also performed a parametric study and proposed a general rule for simulating moderately unstable detonation that the detonation cell structure can be properly resolved if a minimum of 5 grid points are included in the heat-release zone of the corresponding steady ZND detonation wave. On the other hand, Sharpe \& Quirk (2008) found in their resolution study of weakly unstable detonations with regular cells that the resolution of 32 points $/ l_{1 / 2}$ is sufficient for the cellular dynamics and size to be grid-independent. These situations closely resemble the conditions of the present investigation. Based on these studies, a numerical resolution of 20 grids $/ l_{1 / 2}$ of the corresponding overdriven detonation is therefore considered in this work to investigate the unstable behavior of the oblique detonation waves. Although this grid resolution is higher than has generally been used in previous papers on oblique detonation simulations, a resolution study is required in order to verify the resolution required to obtain acceptably converged results. Results of this resolution study will be discussed in a later section.

Figure 2 shows the temperature contours of oblique detonation waves with different overdrive degrees, induced by the wedge angle $\theta=30^{\circ}, 27^{\circ}, 24^{\circ}$, respectively. In the simulations, inflow conditions are fixed at the free-stream values in both the left and upper boundaries of the domain. Outflow conditions are extrapolated from the interior are implemented on the right and lower boundaries before the wedge. Slip boundary conditions are used on the wedge surface, which starts from $x=0.1$ on the lower boundary. Initially the whole flow field has uniform density, velocity, and pressure, which are calculated according to the incident Mach number $M$ and wedge angle $\theta$.

Numerical results show that there are significant differences on the detonation surface depending on the initial and mixture conditions. With high overdrive degree of 2.37 , the surface of the oblique detonation is smooth before $x=40.0$. We observe the onset of disturbance after $x=40.0$ and the first clear triple point formation around $x=48.0$. In 

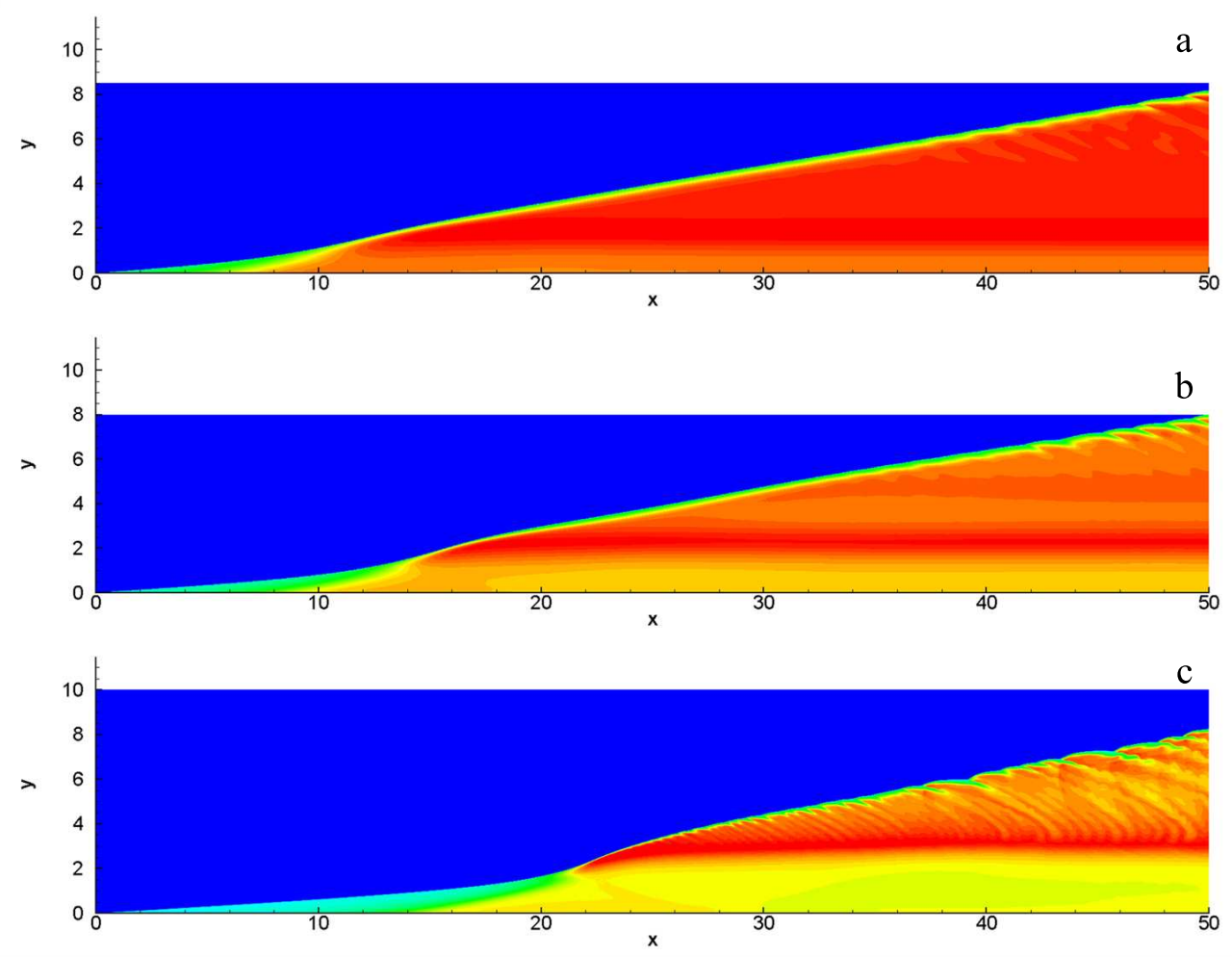

FIgure 2. Temperature flow fields of oblique detonations with $M=15$ and different overdrive degrees: (a) $\theta=30^{\circ}, f=2.37$; (b) $\theta=27^{\circ}, f=2.06$; (c) $\theta=24^{\circ}, f=1.77$.

contrast, with lower degree of overdrive $f=2.06$ the oblique detonation surface becomes unstable earlier approximately at $x=43.0$. Despite the same computation region, we can observe more triple points in figure $2 \mathrm{~b}$ than figure $2 \mathrm{a}$, demonstrating the high overdrive degree suppresses the formation of cellular structures. This is illustrated further in figure $2 \mathrm{c}$, whose surface becomes rapidly cellular with overdrive degree of 1.77. Different from the last two cases, there is no existence of a smooth surface. This data suggests that oblique detonations are stable with large value of $f$ and unstable with the decrease of the overdrive parameter. These results are in agreement with previous conclusions (He \& Lee 1995; Choi et al. 2007; Verreault et al. 2013). We observe, however, that the initially smooth surface eventually becomes unstable with $f=2.06$ and 2.37. In other words, instability is manifested and the onset of cellular structures is eventually observed also in these cases of overdrive degrees above 2.0. This result differs from the previous studies, which suggest the overdrive degree for the stability boundary is just slightly below 1.8, a value close to the stability boundary for one-dimensional normal detonation (i.e., $f=1.73$ ) (Grismer \& Powers 1996; Verreault et al. 2013).

In fact, it is not surprising to see that oblique detonations may be unstable at an overdrive $f$ higher than $\sim 1.8$ previously suggested as the stability boundary for oblique detonation waves. It is worth noting that the result of this study is in fact in agreement with the two-dimensional linear stability analysis of normal cellular detonations. For instance, using the normal-mode analysis approach, Short \& Stewart (1998) show that even at high degree of overdrive $f$, i.e., a value larger than stability limit of $f=1.73$ above which the one-dimensional longitudinal instability is suppressed, a finite band of 

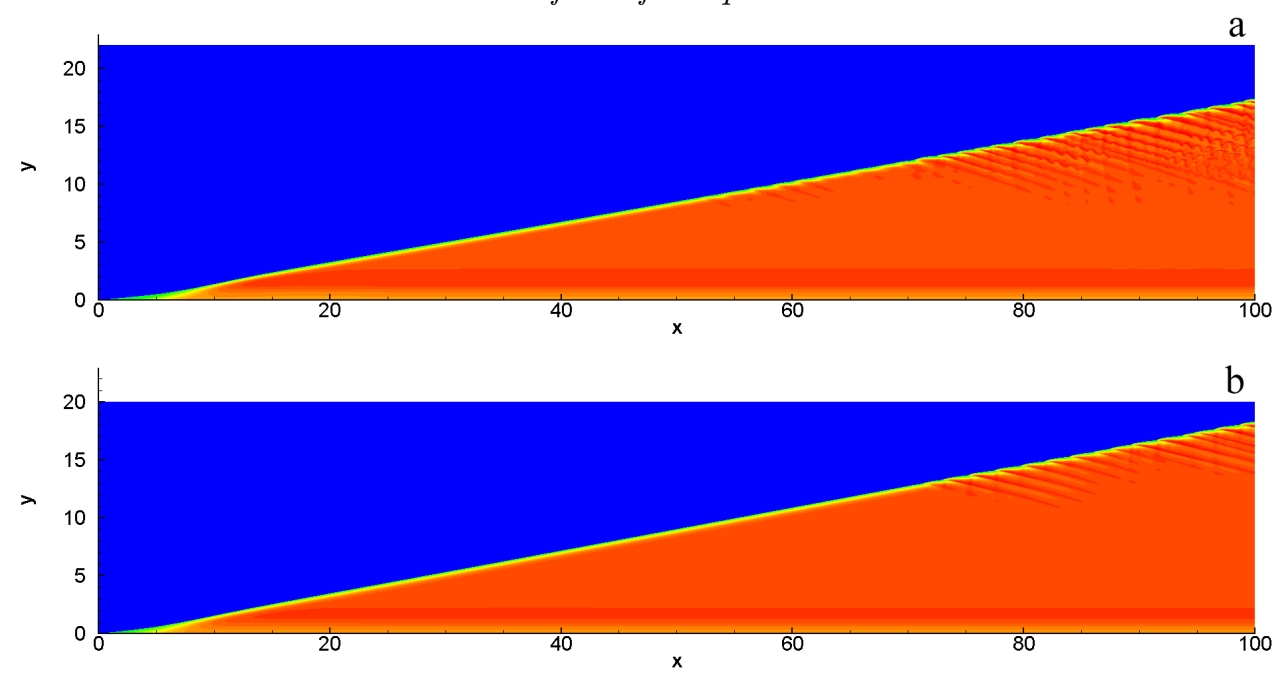

Figure 3. Temperature flow fields of oblique detonations with $M=15$ and different overdrive degrees: (a) $\theta=33^{\circ}, f=2.71$; (b) $\theta=36^{\circ}, f=3.07$.

two-dimensional instability can still persist. For moderate heat release values, increases in detonation overdrive $f$ cannot guarantee stability of the steady detonation wave to transverse linear disturbances, which can be excited to originate the detonation cell formation (Short \& Stewart 1998). Numerical simulations also demonstrate that with sufficient resolution, regular detonation cells can still emerge at very high overdrive (Bourlioux \& Majda 1992).

In order to confirm whether a neutral stability boundary can be obtained, the overdrive $f$ is further increased to 2.71 and 3.07 by using a wedge angle of $33^{\circ}$ and $36^{\circ}$, respectively. Figure 3 shows the results of these very high degrees of overdrive. It is important to note that for these simulation results, the width of the computational domain was increased twofold. Clearly, for both cases, the unstable surface eventually emerges at a later distance. It must be noted that without considering a larger computational width, the unstable surface may not be revealed.

Making again an inference from the study of Short \& Stewart (1998) and as evidenced by these results of very high degrees of overdrive shown in figure 3 , it is in fact unlikely that a stability boundary can be identified for the present mixture. In contrast to previous studies (Grismer \& Powers 1996; Verreault et al. 2013) which suggest a neutral stability boundary of oblique detonation waves for this present mixture of interest to be around $f \sim 1.77-1.79$ close to the 1-D neutral stability boundary of pulsating detonations, the present study shows that, in agreement with the related linear analysis of gaseous cellular detonations (Short \& Stewart 1998), the limit $f \gg 1$ for the present mixture $E_{a}=50$, $Q=50$ and $\gamma=1.2$ is insufficient in itself to render the detonation stable to twodimensional linear disturbances. It may be worth noting that, although neutral stability boundary of oblique detonations are shown to exist for cases with sufficiently small or zero-activation energy limit and small heat of reaction, e.g., Powers \& Aslam (2006), very long time detonation evolution combined with the very high resolution are required to obtain numerically the stability boundary, which remains computationally prohibitive. 

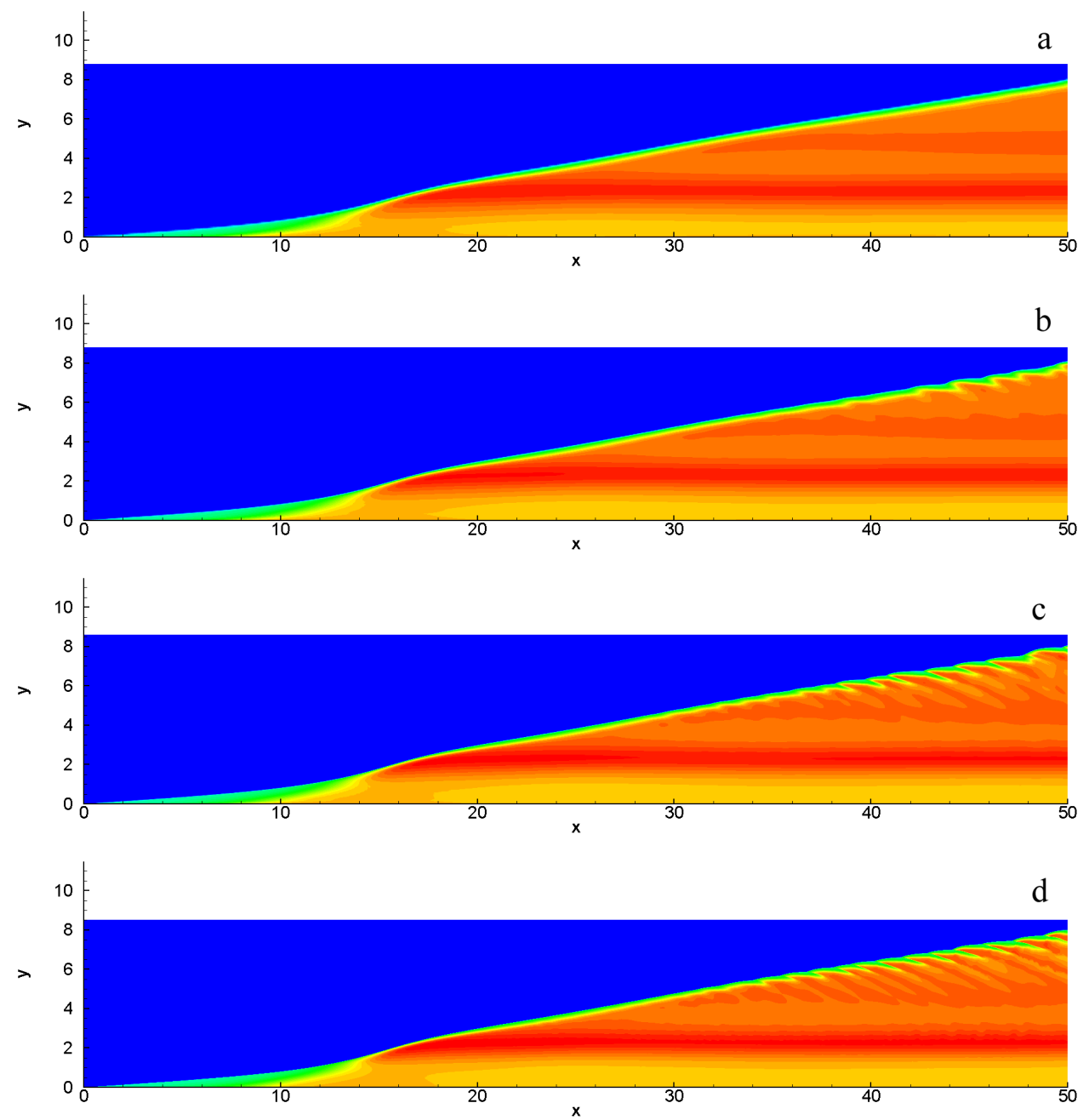

FiguRE 4. Temperature flow fields of oblique detonations with $M=15$ and overdrive degrees $f=2.06$ : (a) grid 0.080; (b) grid 0.040; (c) grid 0.020; (d) grid 0.005 .

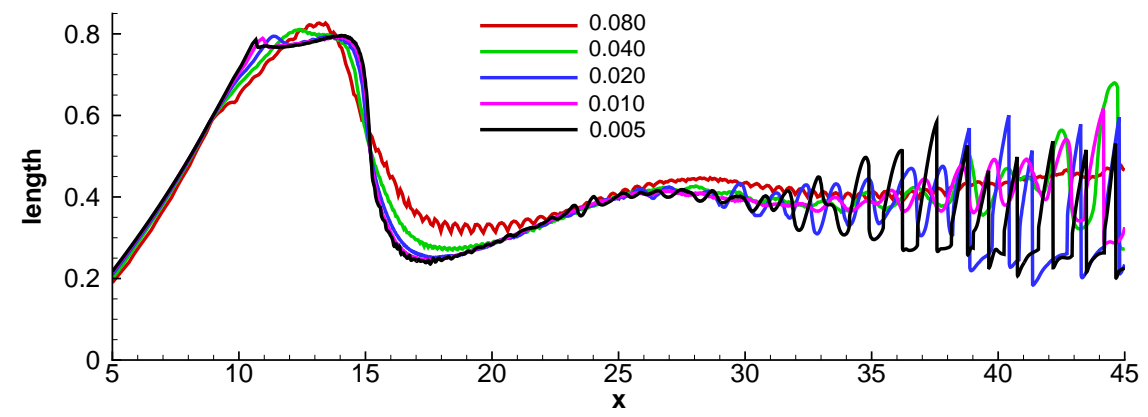

Figure 5. Reaction zone length of oblique detonations with $M=15$ and overdrive degrees $f=2.06$. 


\subsection{Resolution study}

In order to clarify the effect of numerical grid resolution, detonation simulations with different grid sizes are carried out using the same initial and mixture conditions. For instance, figure 4 shows the results with overdrive $f=2.06$ and four different grids of $0.080,0.040,0.020$ and 0.005 , equivalent to $2,5,10$ and 40 points per $l_{1 / 2}$, respectively. Using a grid size above 0.040 , the surface is relatively smooth and any disturbance is hardly noticeable. By reducing the grid scale from 0.040, the disturbance starts to emerge. The result with the smallest grid size of 0.005 shows similar phenomenon as illustrated in figure $4 \mathrm{c}$, although the detailed structure cannot be compared directly due to the randomness of the disturbances. Generally a grid size of 0.020 is sufficient to produce reasonably resolved results, and the difference with further decrease of grid size is hard to distinguish in the flow field. Hence, this resolution study confirms a grid size of 0.010 (i.e., a resolution of 20 points $/ l_{1 / 2}$ ) used in this investigation should provide reasonably converged results to support the observation discussed in section 3.1.

For the investigation of unstable detonations, more strict requirement in terms of numerical accuracy may be necessary. Therefore, the variation of reaction zone length as a function of $x$-position is used as a way to compare the flow fields obtained with different grid sizes and hence, to analyze the effect of numerical resolution. The reaction zone length is defined as the length between the location of $\lambda=0.05$ to $\lambda=0.95$, along the line parallel with the $y$-axis. Equivalently, this value represents the projection of real reaction zone length on the vertical direction. Using this approach, the disturbance of reaction zone can be magnified considerably.

Figure 5 shows the reaction zone length with different grid sizes. All profiles almost overlap together, except two regions where significant differences can be observed. The first region corresponds to the onset of instability leading to the formation of cellular structure, starting from approximately $x=30$; and the second region is the shock to detonation transition occurring between $x=10$ and $x=20$. In the first region, with sufficient resolution the cellular structure evolves from random disturbances. It is observed that for the lowest grid size of 0.080 considered in this test, no noticeable unstable behavior is revealed. However, the use of this very coarse grid gives rise to spurious small oscillations at the beginning after the shock to detonation transition which gradually damp out. These high frequency oscillations are absent for higher grid resolutions and thus, should not be interpreted as physical characteristics of the phenomenon. For all other grid size cases, the origin of unstable behavior is located almost at the same position except for the lowest grid size of 0.040. It is noticed that the grid size of 0.010 has the smallest average amplitude compared with the grids sizes of 0.020 and 0.005 . Due to the random disturbances in this strongly nonlinear system, we cannot yet interpret its mechanism. However, these results demonstrate that the formation of the unstable detonation surfaces is independent of the grid, provided sufficient resolution is used. In the second region the reaction can also be very sensitive to the numerical resolution due to the high temperature flow fields. Nevertheless, it is observed that the profiles with grid size of 0.020 are already very close to the profiles with even finer grids, and the profiles with grid sizes of 0.010 and 0.005 are almost the same.

Considering the above resolution study, all subsequent simulations are therefore fixed with the grid size of 0.010 to investigate the unstable surface of oblique detonations. In fact, the present resolution study is helpful to understand why the instability boundary is found in previous studies at lower degree of overdrive. For example, in the study by Verreault et al. (2013), only about 13 grids are used in half reaction zone length of the corresponding overdriven detonation when $\theta=24^{\circ}$, and one can deduce that there are 

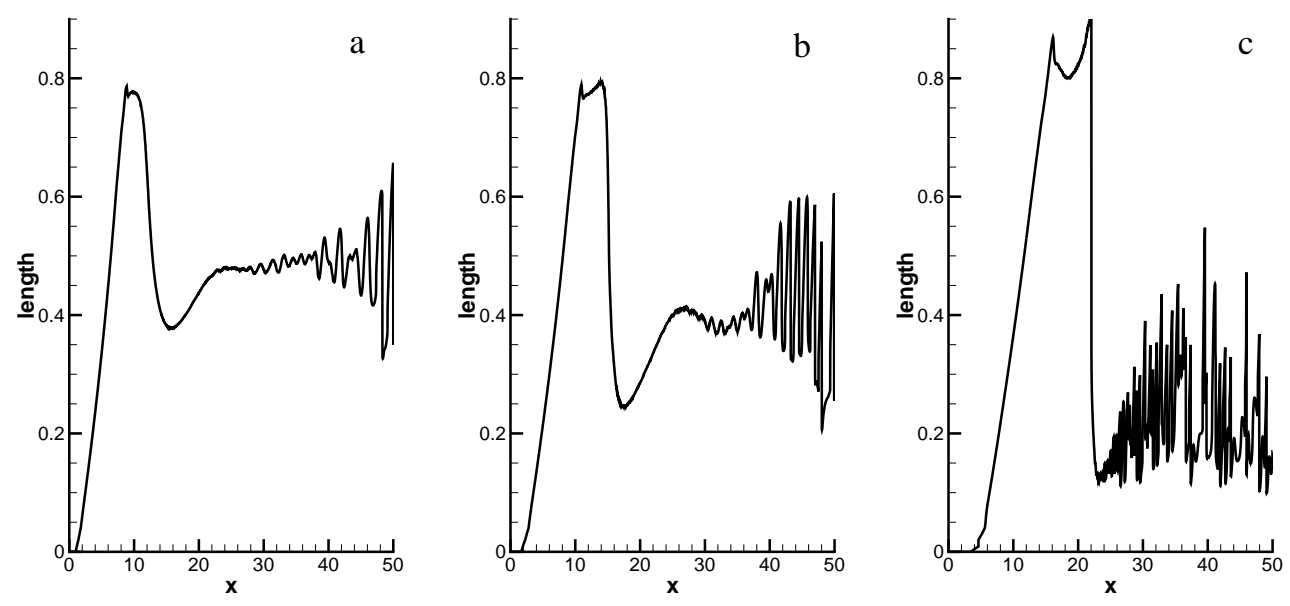

Figure 6 . Reaction zone length of oblique detonations with $M=15$ : (a) $\theta=30^{\circ}, f=2.37$; (b) $\theta=27^{\circ}, f=2.06$; (c) $\theta=24^{\circ}, f=1.77$.

much less grids when $\theta$ rises without either adjusting the pre-exponential factors $k$ or reducing the grid size for finer spatial scales. A poor grid resolution may induce numerical smearing which suppresses the development of instability and delay the cellular structure formation. In this study, a higher resolution is considered by using the grid size of 0.010 and adjusting the pre-exponential factor $k$ in the reaction model as to give a resolution of 20 grids in the half reaction zone of the overdriven detonation. The equivalent resolutions for a normal CJ detonation are 298 grids in half reaction zone length for the case with $\theta=24^{\circ}$ and $f=1.77$, while 806 grids are obtained for $\theta=30^{\circ}$ and $f=2.37$. Thus one must be careful on how to properly define and interpret a sufficient spatial resolution as demonstrated by this large grid resolution difference between CJ detonations and oblique overdriven detonations.

\subsection{Analysis of the reaction zone length}

Figure 6 shows the profiles of reaction zone length in different cases, corresponding to the flow fields shown in figure 2. The profiles are similar at the initial stage, but different from each other after $x=20.0$. Initially the reaction length rises, and subsequently reaches its maximum value before the shock to detonation transition. The transition makes this length scale decrease rapidly to the minimum value, but shortly after it increases again in a gradual manner. For the case of high overdrive degree of 2.37 , the reaction zone length reaches approximately a constant value and then disturbances begin to manifest to form large oscillations, see figure 6a. With moderate overdrive degree of 2.06 , the reaction zone length varies first with a low frequency oscillation and subsequently embedded with higher frequency disturbances. Finally with low overdrive degree 1.77, the oscillations appear just after the shock to detonation transition and the oscillations appear to be around an average value of the reaction length. We must stress here these oscillations are physical rather than numerical, extracted from the flow field shown in figure 2. These may seem like profiles resulting from numerical artifacts, however, it is worth pointing out that the flow field in $x$-direction is indeed compressed dramatically. Comparing these three profiles shown in figure 6, a significant difference in the third one from the other two can be seen. The profile becomes cellular immediately after the shock to detonation transition, and so there is no smooth surface on the oblique detonation. Generally, two 


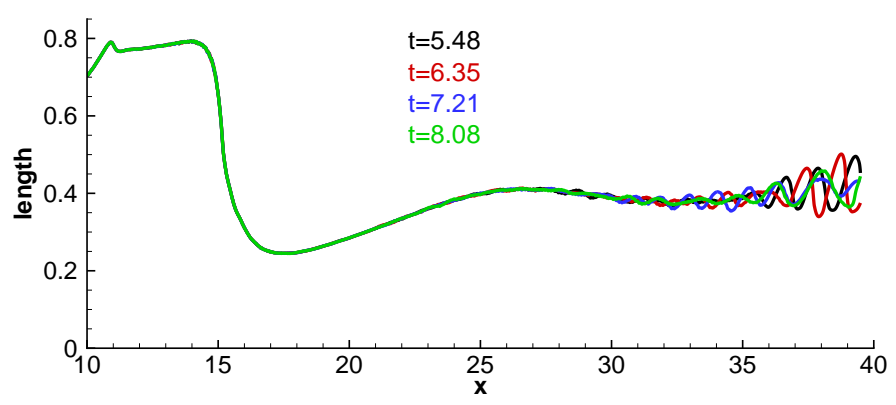

Figure 7. Unstationary length of reaction zone with $M=15$ and $f=2.06$.
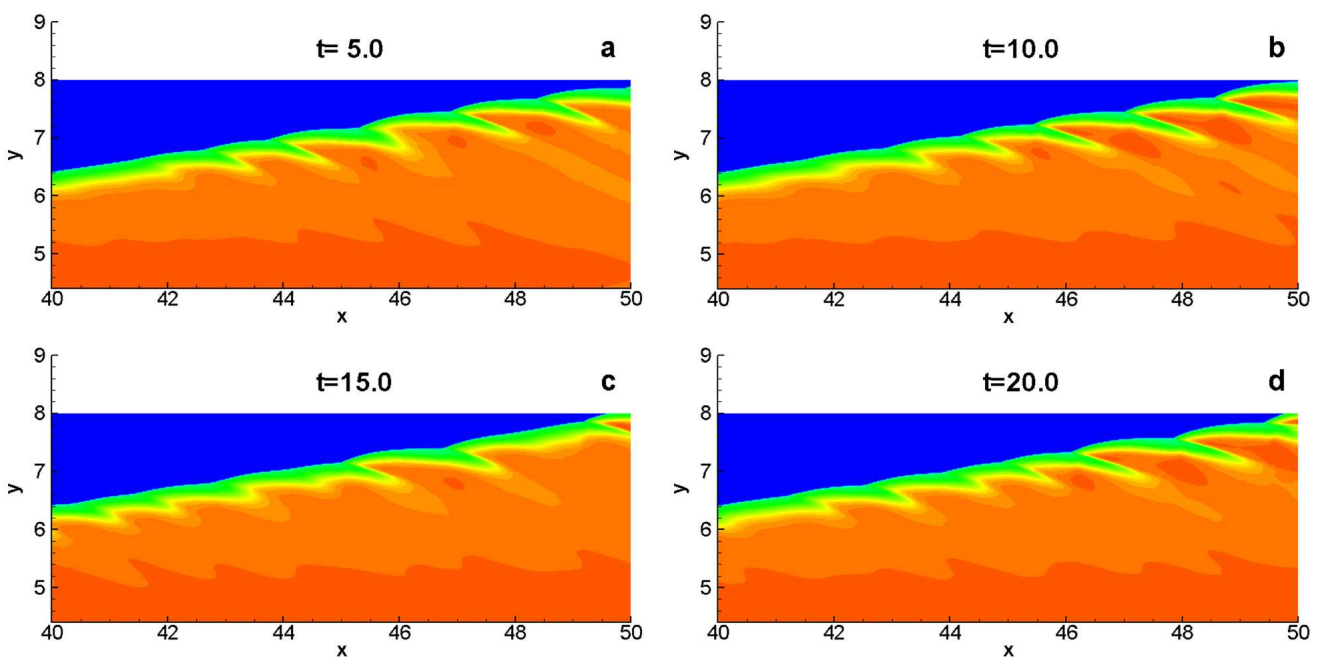

Figure 8. Temperature flow fields of cellular structures with $M=15$ and $f=2.06$.

types of oblique detonations are observed, i.e., one is weakly unstable characterized by the existence of a planar smooth surface, while the other is unstable characterized by immediate manifestation of instability leading to the formation of a cellular structure.

It is interesting to note that even for weakly unstable detonation, the cellular structure is found to be non-stationary or unsteady. The length profiles shown in figures 2 and 6 correspond to a snapshot at a non-dimensional time of 8.52. Figure 7 shows profiles with $f=2.06$ at four earlier time instances with an almost identical time-step increment. Small disturbances come from the upstream, and then amplify in the region behind the crest point. However, defining the point of origin where the length becomes unstable is impossible. The oscillation appears to be random and difficult to predict, similar to cellular structure evolution in normal detonations.

Figure 8 shows the evolution of the cellular detonation structure at a longer time, from $t=5.0$ to 20.0. At the initial transient, the chemical reaction occurs on the wedge, and then the deflection angle of the oblique detonation rises gradually. The oblique detonation reaches its final position before $t=5.0$, and at this time the cellular structures have also appeared on the surface, as shown in figure 8a. It is found that these cellular structures developed from the frontal instabilities continue to generate and travel downstream outside the computational domain. In the present simulation, there is no sign of decaying of these instabilities features even until a much later computational time of $t=20.0$. 

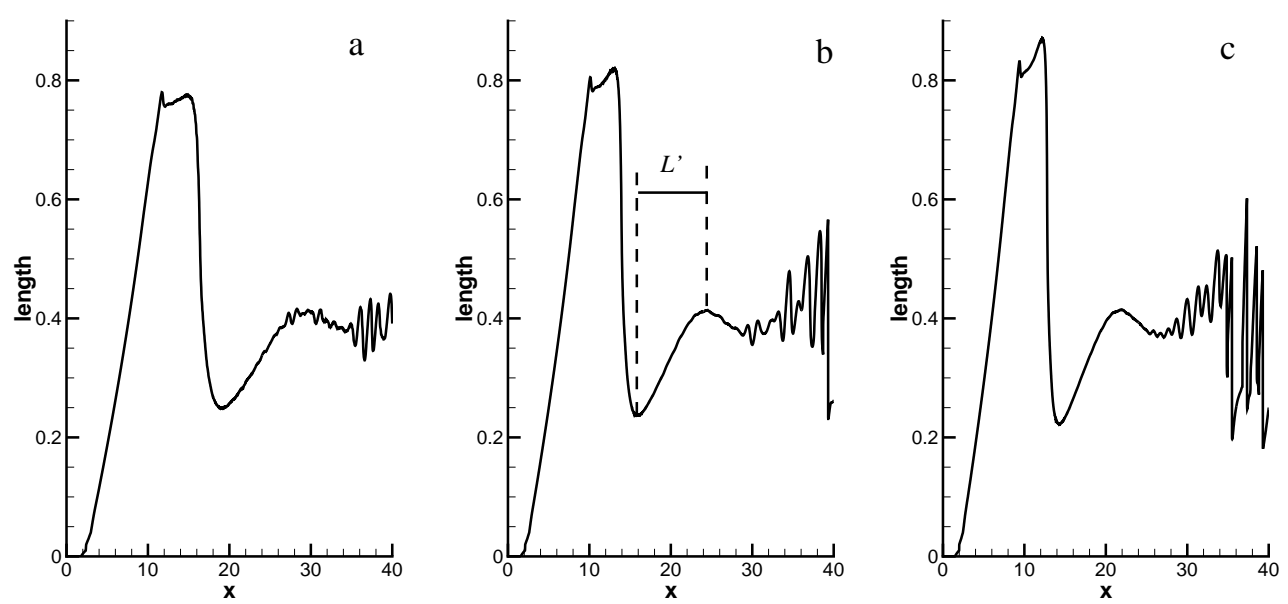

FiguRE 9. Reaction zone length of oblique detonations with $f=2.06$ : (a) $M=16$; (b) $M=14 ;$ (c) $M=13$.

\subsection{Discussion on a stability length}

The aforementioned results show that the oblique surface becomes unstable due to the amplification of disturbances originating upstream, so that the incident Mach number $M$ may influence the destabilizing process. Previous study also demonstrated that detonations become unstable when $M$ decreases at a given fixed wedge angle (Figueira da Silva \& Deshaies 2000). However this conjecture is derived from the consideration of the corresponding change in overdrive degree $f$, and it is still unclear how the incident Mach number $M$ influences the destabilizing process given a fixed overdrive degree $f$. To fix the overdrive degree $f$ while varying the incident number $M$, the wedge angle $\theta$ must be adjusted. In order to start the numerical simulation, the oblique detonation angle $\beta$ is first computed according to Eq. 3.2, and then the wedge angle $\theta$ is obtained according to Eq. 3.1. The incident Mach number $M$ and the deduced wedge angle $\theta$ are thus used to study the effect of incoming flow with different Mach numbers at a given overdrive degree $f$.

Figure 9 shows the reaction zone length with different incident Mach numbers $M$ in the case of $f=2.06$. These reaction zone length profiles are similar to those shown earlier in figure $6 \mathrm{~b}$. In each case, there is an initial increase in the reaction length, which then decreases after the shock to detonation transition. The subsequent relaxation induces one crest, and the disturbances always manifest and amplify behind the crest. Once again, it is difficult to define the exact location of the onset of instability due to the unsteady nature of the structure. Nevertheless, the crest created after the shock to detonation transition can be used as an internal length scale for instability. In fact, it is worth noting that the crest also exists with large overdrive $f=2.37$ as shown in figure 6 a, although the successive decrease is very weak. Therefore, it is possible to introduce a characteristic stability length $L^{\prime}$, defined as the length from the trough to the crest, as indicated in Fig. 6b. Projecting this length $L^{\prime}$ to the oblique wave direction, the stability length $L$ along the surface becomes:

$$
L=\frac{L^{\prime}}{\cos (\beta-\theta)}
$$




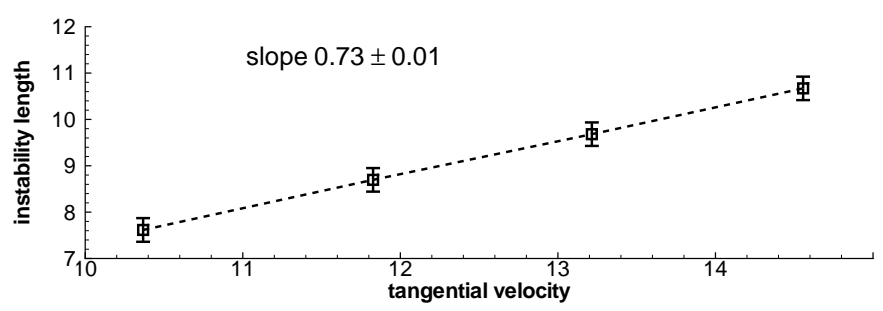

FIGURE 10. Stability length as a function of tangential velocity with overdrive degree $f=2.06$.

$\begin{array}{ccccc} & M=13 & M=14 & M=15 & M=16 \\ f=2.06 & 0.735 & 0.735 & 0.733 & 0.732 \\ f=2.16 & 0.706 & 0.705 & 0.696 & 0.706 \\ f=2.26 & 0.708 & 0.709 & 0.706 & 0.700\end{array}$

TABLE 1. Interior time $\tau$ with different $f$ and $M$.

This length is used here to evaluate the surface instability approximately. Using the same overdrive degree 2.06, $L$ is not a constant, but increases with incident Mach number $M$. Further analysis shows that this length is not only dependent on incident Mach number $M$, but also scaled with the tangential velocity. For instance, figure 10 shows this defined stability length $L$ as a function of tangential velocity, calculated by $U=U_{0} \cos \beta$ with $U_{0}$ as the incident velocity. The stability length is found to be proportional to the tangential velocity, with the slope value equal to $0.73 \pm 0.01$. This demonstrates that the tangential velocity with respect to an oblique detonation is a convective velocity, which only changes the position of unstable surface formation. The line slope, derived from the stability length divided by the tangential velocity, can be defined as the interior time $\tau$. Hence, for a given overdrive degree $f$, there appears only one unique value of $\tau$. This characteristic time scale may thus provide an alternate method to evaluate the oblique detonation instability.

To examine whether the characteristic time $\tau$ defined in this study is universal for different overdrive degrees $f$, additional simulations are performed. With $M=15$, overdrive degrees become $1.96,2.16,2.26$, and 2.37 , corresponding to $\theta=26^{\circ}, 28^{\circ}, 29^{\circ}$, and $30^{\circ}$. For each overdrive degree, we deduce the wedge angle with various $M$ ranging from 16 to 13 , and use these conditions to simulate the oblique detonations. Simulation results show that with $f=1.96$, the disturbance amplifies before the crest. This brings much uncertainties on the measurement of the instability length. This demonstrates the instability length may not be a proper parameter for relatively strong unstable detonations. Nevertheless, other results are shown in Table 1. These results indicate that given the overdrive degrees of 2.16 and 2.26, the stability length is also found to be proportional to the tangential velocity, suggesting the interior time $\tau$ does exist. However, the interior time $\tau$ value becomes approximately 0.70 , a little smaller than 0.73 . This demonstrates that the interior time $\tau$ defined in this investigation is not universal, although it is found to be independent on the incident Mach number $M$. These results suggest more studies are necessary to look for a universal criterion independent of $f$. 


\section{Conclusion}

This paper presents a numerical study on unstable surfaces of oblique detonations using a one-step irreversible reaction model. The detonation polar analysis is used to determine the overdrive degree $f$, and the ZND structures of overdriven detonations are integrated to determine the pre-exponential factor $k$ for the reaction length scale $l_{1 / 2}$ so that the chosen numerical resolution is achieved. This procedure allows the oblique detonation surfaces to be resolved for all cases at the same level on a computational domain with fixed number of grids and provides the basis of the present quantitative analysis. A resolution study as required in any numerical work is also included in this paper to validate the suitability of the chosen numerical resolution used for the simulations.

Results obtained from the present simulations show two types of oblique detonations. One is weakly unstable characterized by the existence of a planer surface, while the other is strongly unstable characterized by an instant manifestation of instability which leads to the formation of a cellular structure. In contrary to previous studies which show that the critical overdrive degree defining the instability boundary is approximately 1.8 , the present result demonstrates that weakly unstable detonations may appear even with high overdrive degree, up to 2.37 considered in this work. This appears to be in accord with results obtained from the two-dimensional linear instability analysis of normal cellular detonations in literature, which shows that even at a large degree of overdrive $f$, higher than the value corresponding to the 1-D neutral stability of normal pulsating detonation $(f=1.73)$, a finite band of transverse disturbances can still be excited to underlie the detonation cell formation as observed in the present investigation. This contradiction between the present results with the published literature could be explained by the low and possibly insufficient resolution used in previous published results. The results from the resolution study show that disturbances can be smeared or not captured by inadequate numerical resolution.

In this work a resolution of 20 grids/hrl of the corresponding overdriven detonation is used thorough which gives relatively better resolution compared to most previous studies on oblique detonations, e.g., Li et al. (1994); Choi et al. (2007); Verreault et al. (2013). It provides reasonably resolved results as verified from the resolution study. However, it should not be concluded that this level of resolution is sufficient for obtaining quantitative values and suitable for all unstable detonation simulations. For cases with highly irregular detonation cells and for numerical studies aiming to predict the correct quantitative value of the growth rate, frequencies, cell spacing, stability boundary, etc., much higher resolutions are likely to be required (Short \& Stewart 1998; Sharpe \& Falle 2000; Sharpe \& Quirk 2008; Taylor et al. 2009). In fact, for very unstable detonations with highly irregular structure, the notion of "grids per reaction zone length", i.e., pts $/ l_{1 / 2}$, should also be carefully interpreted. Having a sufficient number of points within the wavelength of a high frequency unstable mode in highly unstable detonations or equivalently, using a sufficient spatial resolution within the fine length scales in the modeling of the multiscale nature of detonations particularly with detailed chemistry can be a more influential factor for the accurate computation of the phenomenon (Powers 2006; Powers \& Aslam 2006).

Analyzing the present results, a characteristic length of unstable surfaces is defined, which is found to be proportional to the tangential velocity. Further analysis leads to the identification of a characteristic interior time $\tau$, which is independent of the incident Mach numbers at a given fixed overdrive degree $f$. Additional simulations show that although there exists one unique interior time $\tau$ independent of $M$ for each overdrive degree, its value changes when the overdrive degree varies. It is also found that the 
proposed interior time $\tau$ cannot represent a stability parameter for strongly unstable detonations, demonstrating more studies are necessary.

In addition, as shown in the recent study by Romick et al. (2012), the effect of viscosity and physical diffusion (with length scales comparable to that of reaction) can influence the location of the neutral stability boundary of 1-D unsteady detonation and its dynamics in the unstable regime. For two-dimensional unstable cellular detonations with highly irregular frontal structure, typically in combustible mixtures with large activation energy or low degree of overdrive, the Euler and Navier-Stokes models can lead to different structures provided the grid is sufficiently refined in the Navier-Stokes calculations so that the physical viscosity comes to dominate the numerical viscosity Powers (2006); Mazaheri et al. (2012). It may be of interest to carry over these findings to the oblique detonation studies, yet an even much higher resolution than 20 grids $/ \mathrm{hrl}$ is undoubtedly necessary to resolve both diffusion and viscous length scales apart from that of the chemical reaction in order to capture the correct dynamics of the phenomenon.

Acknowledgements: The research is supported by The National Natural Science Foundation of China NSFC No.11372333 and No.90916028; and the Natural Sciences and Engineering Research Council of Canada (NSERC).

\section{REFERENCES}

Bourlioux, A. \& MAJdA, A. J. 1992 Theoretical and numerical structure for unstable twodimensional detonations. Combust. Flame 90, 211-229.

Bourlioux, A., MajdA, A. J. \& Roytburd, V. 1991 Theoretical and numerical structure for unstable one-dimensional detonations. SIAM J. Appl. Math. 51, 303-343.

Choi, J. Y., MA, F. H. \& YAng, V. 2008 Some Numerical Issues on Simulation of Detonation Cell Structures. Combust. Expl. Shock Waves 44, 560-578.

Choi, J. Y., Kim, D. W., Jeung, I. S., MA, F. \& YAng, V. 2007 Cell-like structure of unstable oblique detonation wave from high-resolution numerical simulation. Proc. Combust. Inst. 31, 2473-2480.

Choi, J. Y., Shin, E. J. \& Jeung, I. S. 2009 Unstable combustion induced by oblique shock waves at the non-attaching condition of the oblique detonation wave. Proc. Combust. Inst. 32, 2387-2396.

Figueira DA Silva, L. \& Deshaies, B. 2000 Stabilization of an oblique detonation wave by a wedge: a parametric numerical study. Combust. Flame 121, 152-166.

Fusina, G., Sislian, J. P., \& Parent, B. 2005 Formation and stability of near chapmanjouguet standing oblique detonation waves . AIAA J. 43, 1591-1604.

Gui, M. Y., FAn, B. C. \& Dong, G. 2011 Periodic oscillation and fine structure of wedgeinduced oblique detonation waves. Acta Mech. Sin. 27, 922-928.

Grismer, M. J.\& Powers, J. M. 1996 Numerical predictions of oblique detonation stability boundaries. Shock Waves 6, 147-156.

He, L.\& LEe, J. H.S. 1995 The dynamical limit of one-dimensional detonation. Phys. Fluids 7, 1151-1158.

Hwang, P., Fedkiw, R. P., Merriman, B., Aslam, T. D., Karagozian, A. R. \& Osher, S. J. 1995 Numerical resolution of pulsating detonation waves. Combust. Theory Modelling 4, 317-240.

Kailasanath, K. 2003 Recent developments in the research on pulse detonation engines. AIAA J. 41, 154-159.

LeE, H. I. \& Stewart, D. S. 1990 Calculation of linear detonation instability: one-dimensional instability of planar detonations. J. Fluid Mech. 216, 103-132.

Li, C., Kailasanath, K. \& Oran, E. S. 1993 Effects of boundary layers on oblique-detonation structures. AIAA Paper 1993-0450.

Li, C., Kailasanath, K. \& Oran, E. S. 1994 Detonation structures behind oblique shocks. Phys. Fluids 6, 1600-1611. 
Mazaheri, K., Mahmoudi, Y. \& Radulescu, M. I. 2012 Diffusion and hydrodynamic instabilities in gaseous detonations. Combust. Flame 159, 2138-2154.

Nettleton, M. A. 2000 The applications of unsteady, multi-dimensional studies of detonation waves to ram accelerators. Shock Waves 10, 9-22.

NG, H. D. And Zhang, F. 2012 Detonation instability. In Shock Wave Science and Technology Reference Library, Vol. 6 (ed. Zhang, F.), pp. 107-212. Springer.

PapaleXAndris, M. V. 2000 Numerical study of wedge-induced detonations. Combust. Flame 120, $526-538$.

Powers, J. M. 2006 Review of multiscale modeling of detonation. J. Propul. Power 22, 12171229.

Powers, J. M. \& Aslam, T. D. 2006 Exact solution for multidimensional compressible reactive flow for verifying numerical algorithms. AIAA J. 44, 337-344.

QuiRK, J. J. 1994 Godunov-type schemes applied to detonation flows.. In In Combustion in High-Speed Flows (ed. Buckmaster, J. et al.), pp. 575-596. Kluwer.

Romick, C. M., Aslam, T. D. \& Powers, J. W. 2012 The effect of diffusion on the dynamics of unsteady detonations. J. Fluid Mech. 699, 453-464.

Roy, G. D., Frolov, S. M. Borisov, A. A. \& Netzer, D. W. 2004 Pulse detonation propulsion: challenges, current status, and future perspective . Prog. Energy Combust. Sci. 30, 545-672.

Sharpe, G. J. 1997 Linear stability of idealized detonations. Proc. R. Soc. Lond. A 453, 26032625.

Sharpe, G. J. \& Falle, S. A. E. G. 1999 One-dimensional numerical simulations of idealized detonations. Proc. R. Soc. Lond. A 455, 1203-1214.

Sharpe, G. J. \& Falle, S. A. E. G. 2000 Numerical simulations of pulsating detonations: I. Nonlinear stability of steady detonations. Combust. Theory Model. 4, 557-574.

Sharpe, G. J. \& Falle, S. A. E. G. 2000 Two-dimensional numerical simulations of idealized detonations. Proc. R. Soc. Lond. A 456, 2081-2100.

Sharpe, G. J. \& Quirk, J. J. 2008 Nonlinear cellular dynamics of the idealized detonation model: Regular cells. Combust. Theory Model. 12, 1-12.

Short, M. \& Quirk, J. J. 1997 On the nonlinear stability and detonability limit of a detonation wave for a model three-step chain-branching reaction. J. Fluid Mech. 339, 89-119.

Short, M. \& Stewart, D. S. 1998 Cellular detonation instability. Part 1. A normal-mode linear analysis. J. Fluid Mech. 368, 229-262.

Taylor, B. D, Kasimov, A. R. \& Stewart, D. S. 2009 Mode selection in weakly unstable two-dimensional detonations. Combust. Theory Model. 13, 973-992.

TEnG, H. H. \& JiAng, Z. L. 2012 On the transition pattern of the oblique detonation structure. J. Fluid Mech. 713, 659-669.

Toro, E. F. 1999 Riemann solvers and numerical methods for fluid dynamics. Springer.

Verreault, J., Higgins, A. J. \& Stowe, R. A. 2013 Formation of transverse waves in oblique detonations. Proc. Combust. Inst. 34, 1913-1920.

Viguier, C., Figueira da Silva, L., Desbordes, D. \& Deshaies, B. 1997 Onset of oblique detonation waves: comparison between experimental and numerical results for hydrogen-air mixtures. Proc. Combust. Inst. 26, 3023-3031.

Viguier, C., Gourara, A., Desbordes, D. \& Deshaies, B. 1999 Three-dimensional structure of stabilization of oblique detonation wave in hypersonic flow. Proc. Combust. Inst. 26, 3023-3031.

Vlasenko, V. V. \& Sabelnikov, V. A. 1995 Numerical simulation of inviscid flows with hydrogen combustion behind shock waves and in detonation waves. Combust. Explos. Shock Waves 31, 376-389. 